\title{
Introduction : Pour une nouvelle problématique du sacrifice
}

Introduction: For a new approach to the problem of sacrifices

Luc de Heusch

\section{(2) OpenEdition}

\section{Journals}

Édition électronique

URL : http://journals.openedition.org/span/269

DOI : $10.4000 /$ span.269

ISSN : 2268-1558

Éditeur

École pratique des hautes études. Sciences humaines

\section{Édition imprimée}

Date de publication : 1 septembre 1976

Pagination : 7-22

ISSN : 0294-7080

\section{Référence électronique}

Luc de Heusch, «Introduction: Pour une nouvelle problématique du sacrifice », Systèmes de pensée en Afrique noire [En ligne], 2 | 1976, mis en ligne le 04 juin 2013, consulté le 22 septembre 2020. URL : http://journals.openedition.org/span/269 ; DOI : https://doi.org/10.4000/span.269 


\section{Introduction}

POUR U NE NOUVELL E

$P R O B L E ́ M A T I Q U E \underset{p a r}{\text { Da }}$

Luc de Heusch

Nous présentons ici la première partie des exposés sur le sacrifice qui furent discutés en 1975 et 1976 au sein du Laboratoire associé 221. Le célèbre essai d'Hubert et Mauss date de 1899 (1). Depuis lors, aucune analyse systématique d'une telle ampleur n'a étê entreprise, en dépit d'une foule de suggestions partielles fécondes et de 1'ampleur des informations nouvelles, concernant ce rite majeur (mis en oeuvre de manière diverse par les sociétés les plus variées). Il nous a paru utile, au cours d'une première phase, d'interroger nos propres matériaux d'enquête avant d'élargir 1 'horizon à d'autres civilisations, grâce au dialogue de 1'anthropologie et de l'histoire (2).

Plusieurs questions théoriques dominent cette relecture collective de I'Essai, que J.-P. Colleyn présente d'entrée de jeu. Existe-t-il dans les ensembles rituels examinés une zone sacrificielle (explicite ou implicite) et quels en sont les traits spécifiques ? Ceux-ci se laissent-ils réduire à un ou plusieurs modèles ? En particulier, le sacrifice peut-il être décrit partout et toujours comme un système de communication distinct de l'offrande, ainsi que l'affirment Hubert et Mauss? Serions-nous victimes d'une illusion sacrificielle, de même nature que l'illusion totémique débusquée par Lévi-Strauss ? EvansPritchard estimait que l'Essai est un chef-d'oeuvre peu

(1) H. HUBERT et M. MAUSS, Essai sur la nature et la fonction du sacrifice (1899), in M. MAUSS, Oewvres, I (Paris, 1968), pp. 193-307.

(2) Nous remercions M. Detienne et $\mathrm{Ch}$. Malamoud d'avoir bien voulu répondre déjà à cet appel ; nous espérons pouvoir publier prochainement leurs contributions éclairantes sur la Grèce et l'Inde. 
convaincant de métaphysique sociologique; il n'en admirait pas moins la tentative de construire une "grammaire du rite sacrificiel" (3).

Les collaborateurs du présent cahier semblent tous admettre, avec une sêrie de nuances, qui devront être approfondies par la suite, la spécificité du sacrifice dans la société qu'ils examinent. Mais il apparaît d'ores et déjà que son organisation symbolique, comme sa finalité religieuse et sociale, présente un champ de variations remarquable. M. Cartry montre que les Gourmantché de Haute-Volta distinguent dans la catégorie générale parli deux types de rites, toujours précédés d'une consultation géomantique : celle-ci prescrit tantôt un padita, c'est-à-dire la mise à mort rituelle d'un ou de plusieurs animaux domestiques, tantôt un padipienti, c'est-à-dire l'offrande d'un oeuf de poule, excluant l'opération précédente. Cartry définit le padipienli comme "un rite sacrificiel sur la scène duquel aucun animal n'est présent" ; la destruction de l'oeuf, appréhendé comme "vie", s'impose néanmoins. On voit immédiatement que cette alternative forme système : à son tour la catégorie padita compose un arbre comportant plusieurs options. Tantôt la victime est "mise dans la bouche" de l'autel bulo (qui matérialise une entité avec laquelle l'animal sera partagé); tantôt au contraire elle est soit jetée soit enterrêe après l'égorgement. Ce champ sémantique est si rigoureux que les Gourmantchê ne considèrent pas comme un vrai parli les "sacrifices" codés par la tradition liturgique, où le choix de la victime ne dépend plus de la machine divinatoire (les cérémonies des prémices, par exemple).

Les Mofu désignent par des termes prẻcis chaque modalité de l'offrande religieuse (toujours accompagnée de prières). J.-F. Vincent décide d'appliquer le terme sacrifice à l'ensemble de ce champ opérationnel. On peut discuter du bien-fondé de cette terminologie ; quoi qu'il en soit, l'auteur montre fort clairement que les Mofu procèdent au découpage systématique des ensembles rituels. La libation ordinaire de la bière aux ancêtres, voire sa "consécration" annuelle pour l'"ouverture du grenier" se laissent aisément distinguer de la mise à mort d'un animal domestique qui implique à son tour une alternative mettant en cause le destinataire : si le rite s'adresse aux ancêtres, le sang doit

(3) E. E. EVANS-PRITCHARD, Theories of primitive Religion (Oxford, 1965), pp. 70-71. 
couler, s'il concerne les génies de la possession, la victime sera étouffée. Cette opposition se répercute dans les modalités de la cuisine rituelle, puisque les participants, unis de manière quasi commnielle à leurs ancêtres, partagent avec eux un repas complet (viande grillée par les hommes, viande préparée en sauce par les femmes, farine de mil et bière) alors que seule la viande de la victime est offerte aux génies de la possession. En outre, il existe chez les Mofu une forme miniaturisée de la cuisine rituelle réservêe aux seuls ancêtres : l'offrande d'une boule de mil et d'un os de chèvre ou de taureau. On voit que le sacrifice aux ancêtres renvoie ici à une cuisine totale ( et totalisante) réunissant les hommes et les femmes, la nourriture végétale et animale, ainsi que les deux modes de cuisson de la viande.

L'importance d'un article ancien de M. Griaule (1940) nous a incités à le reproduire tel quel, bien que les travaux plus récents aient fait progresser considérablement notre connaissance du mécanisme sacrificiel chez les Dogon (4). M. Griaule et G. Dieterlen nous ont montré depuis que la mise à mort et la résurrection du génie de l'eau Nommo, opérées par Dieu lui-même, puis la répétition terrestre de ce sacrifice cosmogonique (le dépeçage du corps du septième ancêtre) constituent le paradigme de tout sacrifice. Je prends le risque de proposer une lecture nouvelle de Dieu d'eau, comme inversion du schéma sacrificiel chrétien et variante du mythe inagine de toutes pièces par Freud dans Totem et Tabou (5).

Voici que se dessine un nouvel horizon symbolique : 1'animal est fortement marqué comme substitut du dieu, de l'ancêtre mythique, voire du sacrifiant lui-même. Le septième ancêtre de la première génération, détenteur de la parole du génie de l'eau Nommo, fut dépecé par ses propres frères; plus tard, l'ancêtre Lebé Serou fut sacrifié à son tour, à la suite d'une rupture d'interdit. Alors le premier sacrifié ressuscita sous la forme du serpent; il déglutit le corps du second sacrifié, s'unissant étroitement à sa personnalité pour vomir les

(4) M. GRIAULE, Remarque sur le mëcanisme du sacrifice dogon (Soudan français), Joumal de la Société des Africanistes, X (1940), pp. 127-130.

(5) "Le sacrifice dogon ou la violence de Dieu". 
pierres d'alliance qui marquent notamment 1 'unitê de la sociêté totémique. Chaque année les Dogon sacrifient une chèvre sur l'autel du Lébé, pour purifier la terre avant les semailles. L'"impur", seul habilité à consommer le foie de la victime, "donne sa propre vie", pour se remplir de celle de 1 'ancêtre et 1 a communiquer ensuite à tous.

Cette partie du mythe qui éclaire l'important culte du Lểé n'est, bien entendu, qu'un aspect d'une démarche infiniment plus complexe; on s'en rendra compte en lisant les commentaires que G. Dieterlen apporte à l'article où $M$. Griaule analyse la redistribution des forces vitales qu'opère tout sacrifice. Cette note introductive nous apporte en particulier de précieuses indications nouvelles sur la fonction purificatrice du sacrifice, son association avec la notion de "souillure", nous y reviendrons.

La configuration symbolique dogon, où la pratique rituelle s'articule à une pensée mythique qui baigne toute entière dans le sang du sacrifice cosmogonique, opérateur de la réorganisation du monde, semble avoir connu d'amples développements dans les civilisations issues du Mandé, elle ne saurait cependant être généralisée à l'ensemble de l'Afrique occidentale. Chez les Gourmantché, le sacrifice divin ou humain (sous sa forme symbolique ou réelle) n'occupe pas plus sur la scène mythique que dans la pratique rituelle cette place privilégiée : la mise à mort rituelle de l'homme était jadis simplement une modalité du padilu (rite impliquant l'égorgement et le rejet de la victime).

La sêquence sacrificielle construite par les Minyanka du Mali s'inscrit dans la catégorie gênêrale du don rituel (kara). Si elle est toujours précédêe d'une offrande préalable d'eau pure et de mil (voire de cendres), $D$. Jonckers insiste cependant sur le fait que l'immolation d'un animal domestique est "l'opêrateur principal d'un rite à caractère magique au cours duquel des forces vitales (nyama) circulent, vêhiculées par le sang". Ph. Jespers, quant à lui, démontre dans un article complémentaire, consacré à 1 'un des cultes particuliers des Minyanka, le Nya, que le sacrificateur manipule des forces cosmiques, par l'intermédiaire d'un système rigoureux de signes graphiques. Inscrits sur une planchette, ils sont en relation directe avec les objets cultuels (les yapere) arrosés du sang des chiens. La visée cosmique du sacrifice caractêrise aussi bien le sacrifice dogon que le sacrifice minyanka, mais elle ne passe pas nécessairement par 
la mise à mort primordiale du dieu ou de l'ancêtre. Chez les Gourmantché, comme chez les Minyanka, tout sacrifice (padipienii ou padita) mobilise une "écriture" ; mais il s'agit cette fois de signes gravés sur un morceau de calebasse et correspondant aux animaux mêmes que $l^{\prime}$ 'on énumère dans la prière. Comme le remarque $M$. Cartry, l'aninal intervient ainsi sous trois modalités complémentaires : comme être de chair et d'os, comme "chose" parlée et comme inscription.

Un champ sacrificiel se laisse donc repérer à l'intêrieur des pratiques rituelles de l'offrande, et parfois au coeur du mythe. Mais les opérations complexes qui s'y dêroulent, pas plus que leurs effets sur l'individu ou la société, ne se laissent aisément réduire à l'unité. Dans leur recherche d'un schème constant, bâti sur celui des rites de passage, Hubert et Mauss se préoccupent sans cesse de ce problème qui n'a pas échappé à leur sagacité. Mais, bien que nuancé, le vocabulaire qu'ils utilisent pour élucider ces différences (sacralisation, dësacralisation, etc.) porte la marque de concepts durkheimiens ambigus. On observera aussi que la purification préalable du sacrifiant, dans laquelle ces auteurs voyaient le premier temps de toute séquence sacrificielle, n'apparaît guère dans le présent recueil que chez les Mofu, mais dans une toute autre perspective que celle des "rites d'entrée" : J.-F. Vincent souligne que la souillure (madana) de la sexualité comme celle de la mort est incompatible avec la participation au sacrifice.

Le modèle mécanique du sacrifice dogon, tel qu'il a été décrit il y a plus de trente ans par M. Griaule (à une époque où ses implications cosmogoniques étaient encore loin d'être connues) postule que la libération d'un ou de plusieurs principes spirituels de la victime déclenche sur 1'autel des échanges énergétiques. Ce modèle, on le retrouve chez les Minyanka et les Songhay. Les spéculations religieuses néoplatoniciennes analysêes ici par A. de Surgy, en jouent apparemment aussi, tout en développant le problème philosophique classique de l'idéalisme, la mise en relation de l'âme et de la matière. Ce premier ensemble mécaniste privilégie l'instance de communication. Mais la contribution de J. Rouch attire immédiatement l'attention sur une singularitê : une même société, les Songhay du Niger, utilise concurremment trois types de sacrifice. Les deux premiers obéissent, au prix de quelques transformations, au modèle décrit par Griaule. En revanche, le troisième consiste en une opération de détournement énergêtique. I1 
s'agit cette fois de voler une âme à Dieu et de crẻer un nouvel autel, en quelque sorte fétichisé, doué d'une force vitale autonome, un toomu. La viande du bélier immolé à cette occasion est impure. Le caractère secret du rite, qui contraste avec la publicitê donnêe aux autres sacrifices, indique que nous avons à faire ici à une appropriation privée (au profit d'une famille ou d'un village) d'une entité spirituelle ; elle s'accomplit au prix d'un véritable court-circuit, excluant du rêseau toutes les puissances transcendantes. Ainsi une démarche typiquement magique s'inscrit au coeur du système religieux.

Or l'idêe que le sacrifice assure aux opérants la mâ̂trise des forces de l'univers, en marge du culte rendu aux ancêtres lignagers directs, est présente à l'esprit des initiés du Nya, en pays minyanka ; les objets yapere arrosés de sang renvoient aux 86 signes fondamentaux de la création. Cette très importante découverte de $\mathrm{Ph}$. Jespers est située dans son contexte sociologique par D. Jonckers qui montre que 1 'utilisation de la formidable puissance des yapere est au fondement de la hiêrarchie sociale; les aînés (les hommes les plus âgés) qui manipulent l'ordre symbolique contrôlent aussi la production et la redistribution des valeurs économiques. Les yapere que les sacrificateurs arrosent de sang se laissent déchiffrer comme "rapports sociaux coagu1és".

Le sacrifice, moyen privilégié de communication avec les dieux, est donc aussi susceptible d'entretenir la puissance d'autels fétichisés, instaurés par 1 'homme. En effet, 1 'institution du Nya est transmissible en dehors des liens familiaux. A l'origine il a été acquis auprès d'un "maîtie de la composition" par un groupe restreint d'individus, en quête de puissance mystique. C'est ainsi que, dans la plupart des villages, chaque quartier dispose d'une centrale énergétique, acquise au loin. Le culte regroupe la majorité des hommes. Et l'autorité traditionnelle des vieillards s'empare de ces signes nouveaux du pouvoir et de la prospérité générale. La machine symbolique est branchée sur 1'univers. Le sacrifice du chien, décrit par Ph. Jespers, fait directement appel aux ancêtres mythiques, organisés selon un ordre hiêrarchique et structural strict. Les premiers jumeaux constituent le moteur central de l'opération sacrificielle ; en effet, ils transforment automatiquement le sang lourd des chiens sacrifiés en un sang très léger qu'ils renvoient vers la victime; celle-ci reçoit alors le 
nyama (la force vitale) du Mya, puissance fétichisée, dont les initiés bênéficient à leur tour en consommant l'animal. C'est la raison pour laquelle le yapere qui correspond dans la jarre sacrificielle au signe des ancêtres jumeaux, "maîtres du $N y a^{\prime}$, est le seul à ne pas recevoir le sang.

Chez les Dogon, comme chez les Minyanka, le sacrifice paraît donc déclencher mécaniquement une redistribution de la force vitale de la victime, dêsignée du même terme (nyama). Les Dogon fondent l'efficacitê de cette opération sur le sacrifice-castration des jumeaux primordiaux, mis à mort par Dieu lui-même pour réorganiser l'univers en désordre. Les Minyanka, en revanche, utilisent souverainement la puissance des ancêtres-jumeaux après s'être approprié au loin les secrets du Nya. Cette démarche n'est pas sans évoquer le détournement magique présenté par Rouch à propos des Songhay. On découvre que des idéologies différentes travaillent une même théorie énergétique, fondamentale en Afrique occidentale.

Les Mofu du Cameroun semblent avoir élaboré un modèle de communication beaucoup plus simple. Indifférents à la puissance symbolique du sang, leur attention se porte sur la cuisine rituelle et le partage des aliments. Le caractère asymêtrique du repas communiel est remarquable: les vivants ont accès à la fois à la part "profane" et à la part "sacrée", mais l'inverse n'est pas vrai, les ancêtres se contentant de celle-ci. Il y aurait lieu probablement de s'interroger sur ce déséquilibre symbolique. Comme les Mofu, un certain nombre de populations bantoues (non examinées ici) semblent déplacer 1 'accent symbolique du sacrifice sur la cuisson rituelle et le partage, soigneusement codifiê, de la victime. Les Thonga du Mozambique, par exemple, assignent aux neveux utêrins (outsiders dans cette société lignagère patrilinéaire) le rôle de sacrificateurs (6). Ceux-ci dérobent rituellement la part de la chèvre sacrificielle rëśervée aux ancêtres dont ils sont les messagers, voire même les représentants. Ils sont tenus de rôtir cette part. "sacrée" sur le chemin du retour, après avoir été chassés du village de leurs parents maternels à coups de boulettes de rumen, tandis que les membres du lignage pour qui ils ont accompli le sacrifice consommeront

(6) H. A. JUNOD, Moeurs et coutumes des Bantous. La vie d'une tribu sud-africaine (Paris, 1936), II, chap. II. 
la part "profane" sous forme de bouil1i. Le sacrifice en tant que cuisine ritualisêe obêit ici à un code sociologique; il exprime les oppositions, voire les contradictions du système social, dans la perspective même qu'indiquaient, mais de façon trop empirique, les travaux de M. Gluckman (7). L'on pourrait montrer que le corps même de la chèvre sacrifiée et partagée est chez les Thonga une image métaphorique de la société toute entière, déchirée par la grande crise du deuil. La position symbolique respective du rumen chez les Thonga et les Mofu attire immédiatement l'attention sur la variation des codes symboliques sacrificiels en Afrique. Cette matière végétale prédigérée, contenue dans l'estomac des ruminants, figure dans les codes culinaire et sociologique mis en oeuvre par les Thonga comme un élément participant du cru, propre à marquer le rejet symbolique des neveux utêrins (dans le rituel de deuil) et des futurs alliés (dans le rituel du mariage). Les Mofu, en revanche, rêpandent le rumen sur 1'aute1 où il assure la liaison des morts et des vivants, en vertu d'une autre propriété symbolique, son imputrescibilité présumée (Vincent).

J.-F. Vincent écarte de la zone sacrificielle mofu la mise à mort rituelle d'un animal effectuée lorsqu'il s'agit de "laver" la souillure (de la mort, de la sexualité). Mais, outre que cette opération rituelle forme système avec le sacrifice communiel en l'honneur des ancêtres (puisqu'il fait appel notamment à des animaux sauvages -- daman, lézard -- en plus du poulet), ces pratiques "purificatrices" nous introduisent au coeur d'une nouvelle problématique générale. Celle-ci est dominante dans le monde nilotique. La "souillure" (madcoma) est "insupportable aux puissances surnaturelles" mofu ; elle doit être arrachêe de temps en temps, comme les toiles d'araignêes de la case "avec la viande d'un animal" qui a pour fonction de "nettoyer madcona". Or les Nuer du Soudan utilisent, mais de façon beaucoup plus complexe, le même type de discours lorsqu'ils commentent l'ensemble des rites sacrificiels individuels. Evans-Pritchard, qui les a admirablement décrits, s'abuse sans doute lorsqu'il suggère d'interpréter le sacrifice nuer dans la perspective du péché (8). Il est clair néanmoins que sa

(7) M. GLUCKMAN, ed., Essays on the Ritual of Social Relations (Manchester/New-York, 1962).

(8) E. E. EVANS-PRITCHARD, Nuer Religion (Oxford, 1956), chap. VIII. 14 
fonction explicite est de débarrasser l'individu sinon d'une "souillure", du moins d'un état spirituel dangereux (dont le signifiant visible est une maladie) ; celui-ci est la conséquence d'une rupture d'interdit. Evans-Pritchard a montré que le sacrifiant s'identifie toujours symboliquement au boeuf ; il l'immole après l'avoir consacré au moyen de la cendre. Nous retrouvons donc ici, mais dans une toute autre perspective, l'un des principes fondamentaux du sacrifice dogon. Les Dogon projettent sur un paradigme mythique (ignorê des Nuer) une idée voisine de l'expiation lorsqu'ils affirment que Dieu a procédé au sacrifice des jumeaux primordiaux pour réorganiser un univers perturbé par la faute du Renard.

La pratique et l'idéologie sacrificielles nuer posent à la thêorie de la communication un nouveau problème ; Hubert et Mauss ne l'ont pas ignoré, mais ils s'efforcent d'en effacer les contours : "Peut-être chercherions-nous en vain des exemples de sacrifices expiatoires où ne se glisse aucun élément communiel ou de sacrifices communiels qui ne ressemblent par aucun côtê à des sacrifices expiatoires" (9). EvansPritchard lui-même s'accroche à cette thèse : selon lui il n'y aurait pas de distinction absolue chez les Nuer entre les sacrifices purificateurs personnels et les sacrifices collectifs, associés aux rites de passage; mais la seule preuve qu'il en administre se limite à quelques lignes : le sacrifice collectif effectué lors du deuil a pour fonction de débarrasser la société de la souillure de la mort. Il n'en est pas moins obligê de sauver les distinctions que sa propre analyse impose avec tant de force, en faisant appel à la terminologie contestable de Hubert et Mauss : les sacrifices collectifs seraient des rites de sacralisation d'un lien social, alors que les rites individuels qui seuls, à vrai dire, mobilisent son attention, seraient des rites de désacralisation, chargés de "débarrasser l'homme de l'Esprit" (10).

Or, la notion ambiguë de désacralisation renvoie ici à la nécessitê de mettre fin à un excès de communication avec les puissances surnaturelles. "Tout grand danger, tout malheur est le signe de l'activité des

(9) H. HUBERT et M. MAUSS, Essai sur la nature et la fonction du sacrifice, op. cit. I, p. 211 .

(10) E. E. EVANS-PRITCHARD, op. cit. p. 198. 
esprits" (11). Cette fois la thêorie cesse de voir dans le sacrifice le moyen de crêer un circuit énergétique; 1 'animal devient au contraire le lieu où s'opère la disjonction de Dieu et de la victime humaine, unis par une conjonction abusive (la plupart des sacrifices expiatoires s'adressent à $1^{\prime}$ Étre suprême).

La notion de "souillure" est manifestement associêe chez les Nuer à un affaiblissement de l'être, provoqué par une intervention pathogène du monde d'en-haut. Or, inversement, les Dogon interprètent le dangereux état physique dans lequel se trouve celui qui a transgressé un interdit comme une perte de substance spirituelle; les plus récentes enquêtes menées par G. Dieterlen ont montré que l'écoulement du sang sacrificiel et le dégagement de force vitale qui l'accompagne ont précisément pour fonction de combler ce "manque". I1 ne s'agit là, à tout prendre, que d'une nouvelle modalité d'un schème constant : purificateur et réorganisateur, le sacrifice intéresse, à des niveaux divers, l'univers, la société et l'individu, menacés, chacun à sa manière, par le désordre. L'harmonie ou l'intégrité compromises doivent être reconstituées par un meurtre rituel libêrant une énergie compensatrice.

M. Cartry met plus radicalement en cause l'universalité d'un modèle fondé sur la théorie de la communication ou sur une interprétation mécaniste. Analysant le système sacrificiel gourmantché, il pressent la nécessité de forger d'autres "outils logiques et topologiques". Il concentre toute son attention sur le champ symbolique qu'institue le monde des "animaux de maison". Deux codes s'entrecroisent ici. Le choix de la victime sacrificielle est déterminé par la "machine divinatoire" opérant sur une liste de base. Celle-ci comporte le poulet, les caprins, les ovins et la pintade; elle exclut le chien, le chat, le cheval, 1'âne; l'individu choisi à l'intérieur de l'une des espèces sacrificielles doit à son tour présenter un certain nombre de caractéristiques. M. Cartry démontre que les animaux sacrifiables et non sacrifiables relèvent respectivement de deux modes différents de relations avec 1'"avoir", et ceci à divers points de vue : statut du propriétaire, mode d'acquisition et d'échange, tendance à l'accumulation, contrôle de la reproduction. Les deux sêries d'animaux domestiques sont hêtêrogènes : les animaux sacrifiables peuvent être traités, à l'inverse des autres, comme pure quantitê, quand bien même la tendance à

(11) E. E. EVANS-PRITCHARD, Zoc. cit. 
1 'accumulation porterait-elle la marque d"une sorte de passion inutile, irréductible à toute visée êconomique fondée sur une recherche de rentabilité". M. Cartry aborde ainsi l'une des questions essentielles du sacrifice : quel est le sens de cette perte, de ce prélèvement sur 1'avoir ? Evoquant Bataille, Cartry observe que la ponction sacrificielle opère sur des animaux réduits "à l'état de chose" ; ceux qui se trouvent exclus du sacrifice, en revanche, "paraissent se déployer dans une sorte de réserve mythique qui aurait étê comme ménagée à 1 'intérieur du monde de l'instrumentalité et de l'activité utilitaire". La pensée mythique explique en revanche comment les vaches, animaux sacrificiels, ont étê soustraites à l'univers aquatique antérieur, pour être culturellement asservies et multipliëes.

La dêsignation et la qualification symboliques des espèces sacrifiables méritent en effet la plus grande attention. Il suffit, pour s'en rendre compte, de comparer le statut du chien chez les Gourmantché et chez les Minyanka. Exclu du sacrifice par les premiers (utilisê seulement par les sorciers), notament parce qu'il n'est jamais un trait de richesse ni une source de profit, le chien se trouve précisément être l'animal sacrificiel par excelience dans le culte minyanka du Nya. Un mythe, commenté par $\mathrm{Ph}$. Jespers, jette les plus vives lueurs sur la position rituelle de cet animal. Les secrets du Nya étaient détenus par des êtres de brousse, descendants d'une première humanité déchue. Au cours d'une partie de chasse, le chien égorgea l'une de ces créatures mythiques et livra les objets du culte aux hommes. Mais il en mourut sur le champ. Or c'est précisément cette mort inexpliquée qui est répétée sans cesse dans le sacrifice, avec une emphase quantitative que souligne, de son côté, D. Jonckers. Ph. Jespers montre que le sacrifice implique d'abord la mise à mort des génies de brousse, intermédiaires entre Dieu et les hommes du village, puis celle du chien qui, participant à la fois de la nature et de la culture, apparaît comme un médiateur privilégié entre leurs habitants respectifs. On enregistre ainsi une sêrie continue de passages entre des univers séparés. Le cycle de la violence sur lequel s'appuie cette transmission des sources mêmes de la vie ne s'arrête pas là puisque l'êtablissement du culte du Mya dans un nouveau village entraîne à plus ou moins longue échéance la mort de celui qui s'en est approprié la puissance. Au terme de ce processus, le sacrifice annuel du chien, rëclamë par le Nya, prend tout 
son sens : il est le substitut atténué de la mise à mort d'êtres mythiques ou rêels, condition nécessaire de $1^{\prime}$ apparition et de l'installation du $N y a$ dans le monde des hommes. Le sacrifice du chien fait en somme figure de dette perpétuelZe, comme si le trop-plein de vie mythique que cet animal introduisit devait être compensê par une perte sensible portant d'abord sur l'être, sur $1^{\prime} \alpha v o i r$ ensuite. Chien ou homme, 1 'initiateur du culte est condamné à une vie raccourcie ; il s'immole en quelque sorte lui-même, car le Nya a été arraché au prix d'un meurtre à ses véritables propriétaires, successeurs déchus de la première humanité, mâ̂tresse de la brousse. La ponction sur $l^{\prime}$ avoir retentit ici sur l'économie. D. Jonckers signale que la plupart des cultes minyanka annuels exigent l'immolation de dix à trente chiens et d'innombrables poulets. Elle insiste sur le surcroit de travail agricole qu'implique cette exigence. Car le chien, contrairement à $1^{\prime}$ option des Gourmantché, est disponible sur le marché.

Expiation de la faute initiale du Renard ou réparation du "manque" instauré dans la personne à la suite d'une rupture d'interdit (Dogon), purification d'une faute actuelle chez les Nuer, dette perpétuelle chez les Minyanka, le sacrifice se réfère à une problématique qui, pour être diverse, n'en porte pas moins la même marque. Chez les Gourmantché enfin, 1'abandon sacrificiel du boeuf (instrument par excellence de stratégie économico-sociale, objet de convoitise arraché au monde mythique de l'eau) restitue, selon la formule de M. Cartry, "quelque chose d'essentiel qui aurait été comme perdu, oublié, amnésié dans la vie quotidienne.

L'ensemble de ces observations partielles suggère que l'animal sacrifiable est fortement chargé de valeurs symboliques. Il importerait d'abord de repérer celles-ci avec précision dans chaque société, avant de reconstituer le champ des variations. On connait assez les liens intimes qui existent entre 1 'homme et son troupeau dans les sociétés pastorales nilotiques. Lévi-Strauss a attiré l'attention sur le jeu des substitutions qu'autorise le sacrifice, en se fondant précisément sur le cas des Nuer (12). I1 y aurait lieu d'examiner attentivement les limites de ce jeu, souvent réglé en Afrique par la machine divinatoire.

(12) C1. LEVI-STRAUSS, La pensée sauvage (Paris, 1962), p. 296. 
Celle-ci ne semble guère intervenir dans le choix de l'animal chez les Nuer. Cependant le sacrifice obéit ici aussi à une codification. Le boeuf est l'animal sacrificiel par excellence; mais par souci de 1imiter les ponctions effectuées sur le troupeau, richesse fondamentale, les pasteurs nuer utilisent plus fréquemment un bélier ou un bouc castrés, qualifiês de "boeufs", par une ruse de langage. La mise à mort varie selon le destinataire et la fonction. Si le sacrifice s'adresse à Dieu, l'animal est transpercé d'une lance sur le côté droit ; s'il s'adresse à un esprit d'en-haut d'origine humaine (mort foudroyé), on utilisera un mode particulier de dépeçage : la victime sera coupée en deux à hauteur du ventre. Si le sacrifice a pour fonction de briser une relation incestueuse, l'irmolation et le dépeçage constituent une seule et même opération : la victime est jetée au sol et partagée en deux, longitudinalement cette fois. Les sacrifices faits à un esprit de I'eau exigent une chèvre ou un mouton, jamais un boeuf ; l'animal est précipité dans la rivière. Inversement, certains rites sociaux particulièrement importants (mariage, fin des hostilités) exigent le sacrifice du boeuf et interdisent toute substitution (13).

Réexaminons dans cette perspective le problème particulier posé par la substitution possible au boeuf d'un fruit sauvage : Cucumis prophetarum (Crowford). En cas de dénuement, cette espèce de concombre couvert de noeuds peut être "sacrifiée", en lieu et place d'un animal. Evans-Pritchard ne nous fournit malheureusement aucune indication qui permettrait de comprendre pourquoi cette plante est le seul représentant du monde végétal (sauvage ou cultivê) à possêder le privilège de "représenter" le boeuf. On observera toutefois qu'elle envahit les champs et qu'on la nome soit kuol soit kwol yang ("concombre" de la vache). L'association symbolique privilégiée avec le bêtail se confirme lorsqu'on examine le rite sacrificiel dont le "concombre" est l'objet. D'un coup de lance, le fruit est coupé en deux parties. La moitié gauche, mauvaise, est jetée au loin tandis que la partie droite, la bonne, est placée sur le chaume de l'enclos du bétail, à l'entrée, après que l'officiant s'est frotté la poitrine et le front avec le jus. La division rituelle VIII.

(13) E. E. EVANS-PRITCHARD, Nuer Religion (Oxford, 1956) chap. 
du fruit êvoque le dêpeçage longitudinal du boeuf effectué dans les sacrifices conjurant les périls de 1 'inceste ; effectivement, EvansPritchard nous apprend que le traitement rituel de Cucumis prophetamm est d'usage courant lorsqu'il s'agit de se purifier de la souillure résultant d'un inceste mineur (14). La position symbolique du concombre sauvage dans sa relation avec le bétail appelle assurément de nouvelles enquêtes chez les Nuer.

Il apparaît aussi que 1e monde des animaux domestiques, objet privilégié des manipulations sacrificielles, ne peut pas être décrit en dehors des relations symboliques qu'il entretient avec le monde des animaux sauvages. Le lézard et le daman font une brusque irruption dans l'autre face (purificatrice) du sacrifice chez les Mofu (Vincent). La mort violente du "singe rouge", de l'hyène, du lièvre, de l'êléphant, du lamentin, apparaissent respectivement au fondement de divers cultes minyanka dans des récits étiologiques qui relatent le passage des yapere (autels fétichisés) de la brousse au village (Jespers). Le nãko (1'autel des chasseurs) reçoit, outre le sang des animaux domestiques, celui de 1 'hyène, de l'oryctéropte, du boa et de l'antilope capturés vivants (Jonckers).

Le problème du sacrifice (en tant qu'immolation d'animaux domestiques) ne saurait être disjoint du problème de la chasse sacrificielle, évoqué déjà, mais trop rapidement, par Hubert et Mauss à propos des Zưni dans un texte qui date de 1906 (15). C'est notamment l'intérêt religieux exclusif apporté aux animaux de la forêt qui permet d'expliquer pourquoi les Lele du Zaĭre ignorent toute forme de sacrifice. Qui plus est, la classification rigoureuse des espèces et 1'opposition radicale introduite par les Lele entre l'homme et 1 'animal, rendent compte du refus singulier de l'élevage des caprins, alors que celui-ci est très répandu chez les Bantous. Les Lele n'ont entrepris l'élevage de la chèvre qu'à une époque récente, sous la pression de la demande commerciale des Luba. En 1950, ils n'abattaient jamais cet animal et se contentaient de l'utiliser pour diverses prestations (16). L'animal est

(14) E. E. EVANS-PRITCHARD, op. cit. p. 203.

(15) H. HUBERT et M. MAUSS, Introduction ã 1 'analyse de quelques phénomènes religieux (1906), in M. MAUSS, Deuvres, I, pp. 3-11. p. 64 .

(16) M. DOUGLAS, The Lele of the Kasai (Londres/Ibaden/Accra, 1963), 
un habitant de la forêt et la présence de la chèvre au village est, aux yeux des Lele, un véritable scandale logique. La viande de la chèvre suscite le dégoût ; 1'"impuretê" (hama) qui s'y attache est, au sens strict, une "impropriêté" symbolique. Exclue ontologiquement de l'univers de l'homme, la chèvre ne participe pas ici du monde de l'avoir, alors qu'elle constitue l'instrument par excellence de la communication avec les ancêtres chez les Thonga, évoqués précédemment. En revanche, les Lele utilisent un animal singulier, le pangolin, qui participe symboliquement à la fois du monde humain (par son mode de reproduction monopare) et du monde animal (où il fait figure de monstre taxinomique), pour assurer la communication, nécessaire et difficile, entre le village et la forêt, où les animaux vivent dans la proximité des esprits (17).

Enfin, le champ sémantique du sacrifice devrait sans doute être étendu parfois à des domaines voisins, là où les spéculations sur le sang dominent la scène rituelle. Chez les Dogon, par exemple, les mythes articulent clairement en un ensemble cohérent sacrifice, circoncision et menstruation. Le problème de la mort est, comme en d'autres lieux sous des formes différentes, au coeur du-sacrifice car le paradigme mythique de la vie brève se laisse déchiffrer ici comme sacrifice manqué, alors que le sacrifice rêussi est, me semble-t-il, une disparition simulée, jouée en vue de la résurrection et de la réconciliation (voir "Le sacrifice dogon ou la violence de Dieu").

Bien d'autres problèmes ont êté évoqués au cours des discussions. Et notamment les rapports complexes (équivalence ou diffērence ?) entre le sacrifice animal et les divers traitements rituels réservês à la graine, en particulier lors des offrandes ordinaires ou extraordinaires (prémices). Des enquêtes précises devraient être entreprises ici encore sur les propriētés symboliques de chaque espèce végétale, et 1a substitution possible de la graine à l'animal. Rappelons que les Dogon rapprochent eux-mêmes le battage du fonio, graine chargée d" "irnpureté", du sacrifice et de la menstruation, dette de sang originelle des femmes à la terre (18).

(17) L. de HEUSCH, préface à M. Douglas, De Za Souizlure (Paris, 1971).

(18) M. GRIAULE, Dieu d'eau. Entretiens avec Ogotemméli (Paris, 1948), pp. $182-183$. 
G. Dieterlen montre ici-même que certains rites agraires (jouant du cru et du cuit) se fondent sur le sacrifice cosmogonique du Nommo : la fermentation du grain, tué par la cuisson, évoque la résurrection du gênie réorganisateur. On voit que la cuisine sacrificielle agit cette fois sur l'ensemble de l'univers.

Ces trop brèves incursions n'ont d'autre ambition que d'éveiller l'intérêt pour de nouveaux parcours, voire pour une nouvelle exploration systématique $d$ 'un phénomène dont l'apparente banalité recouvre une multitude de problèmes symboliques, économiques et sociaux non résolus. Un intêrêt semblable au nôtre s'est manifesté récemment chez les hellénistes et les indianistes. Nous saluons ici notamment les travaux de M. Biardeaux et Ch. Malamoud (19), ceux de J;-P. Vernant. et $M$. Detienne. Le temps d'un dialogue généralisé est sans doute arrivê. Nul lieu n'y est plus propice que la Ve section de l'E.P.H.E.

(19) M. BIARDEAUX et Ch. MALAMOUD, Le sacrifice dans l'Inde ancienne (Paris, 1976). 